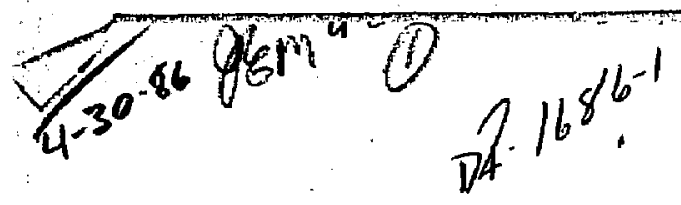

SLAC-PUB--389I

DE86 009781

\section{WAKE FIELD ACCELERATORS *}

\author{
P. B. W'Inson
}

Stanford Linear Accelerator Center

Stanford University, Stanford, Calijornia, 8/905

Invited talk presented at the SLAC Sumıner Institute on

Particle Physics, Stanford, California, July 29 - August 9, 1985
$00 \sqrt{15}-860736-2$

\section{Introduction}

In a wake field accelerator a high current driving bunch injected into a structure or plusma produces intense induced fields, which are in turn used to accelerate a trailing charge or bunch. The driving bunch and the accelerated bunch may or may not follow the same paih through the structure. An important concept is the sa-called transformer ratio. The driving burch induces a retarding field within the burch as the kinetic energy of the bunch is transformed into electromagnetic energy in the wake field. The maximum absolute value of this retarding field is denoted by $E_{m}^{-}$. The transformer ratio is then $R \equiv E_{m}^{+} / E_{m}^{-}$, where $E_{m}^{+}=E_{a}$ is the maximum acrelerating gradient seen by a test charge moving behind the driving bunch on the same or on a parallel path. The retarding and accelerating fielda are assumed to be averaged over the length of the structure, or over one period if the atructure is periodic.

In a bense, almost all accelerators are wake field accelerators. For example, in a conventional rf-driven accelerator a high current, low voltage beam induces an if voltage in the output cavity of a klystron. The induced electromagnetic wave (the wake feld) then travels through a waveguide to the accelerating structure. In this lecturc, however, we consider only wake field acceleration in the conventiona' sense, in which a driving bunch is injected into a metallic cayity or structure. A close relative of this type of wake field accelerator is the plasma wake field accelerator. In this cevice a driving bunch is injected into a plasma, setting up strong longitudinal plasma oscillations which can be used to accelerate a following bunch. The plasma wake field accelerator is treated elsewhere in these proceedings. 1

\footnotetext{
- Work aupported by the Department of Energy, contract DE - ACoj - T6SF00515.
} 


\section{DISCLAIMER}

\section{Basic Concepts}

Consider a point charge $q$ moving in free space with a velocity close to that of light. The electric and magnetic field lines will lie nearly in a transverso plane, with an opening angle given approximately by $1 / \gamma$. Suppose now that the charge moves past a perturbjing metallic obstacle, which is displaced from the path of the charge by a distance $b$ es shown in Fig. 1 , and that it passes the point $z=0$ at $t=0$. Scattered radiation will tend to fill in behind an expanding spherical wavefront, traveling at the velocity of light, as shown sehernatically in the figure. Suppose a test particle follows the same path along the $z$ axis as the driving charge $q$, but at a fixed distance $s$ behind. At time $t \approx\left(b^{2}+s^{2}\right) / 2 c s$, the scattered field will begin to reach the axis at position $z_{\mathrm{f}}=z_{q}-5$ producing both longitudinal and transverse forces acting on the test particle. The intensity of the forces due to these "wake fields" is proportional to the magnitude of the driving charge. Furthermore, the wake field forces can be greatly enhanced by entirely surrounding the axis with an appropriately shaped metallic boundary. The driving charge can in addition be a shaped charge distribution, and the test charge need not follow the same path as the driving charge.

The goal of wake field accelerator design is to adjust the structure geometry, the shape of the driving charge distribution and the paths of the driving and accelerated charges to strike an optimum balance among the following four quantities:

$$
\begin{aligned}
R & \equiv \frac{E_{a}}{E_{m}^{-}} \\
\eta & =\frac{u}{q E_{m}^{-}} \\
k_{\ell} & \equiv \frac{E_{a}^{2}}{4 u} \\
k_{a} & \equiv \frac{E_{a}}{2 q}=\frac{2 \eta k_{\ell}}{R} .
\end{aligned}
$$

This report was prepared as an account of work sponsored by an agency of the Uniced States Government. Neithes the United States Government nor any agency thereof, nor any of their cmployces, makes any warranty, express or implied, or assumes any legal liabifity or cesponsibility for the accuracy, completeness, or uscfulness of any informotion liabifty or tesponsiprocess disclosed, or represents that its usc would of any information, apparatus, product, or ence herein to any represents that its nse would not infringe privitely owned rights. Refermanufaciurer, or atherwice commercial product, process, or service by trade name, trademark, manufacturer, or atherwise toes not necessarily constitute or imply its endorsement, recommendation, or favoring by the United States Government or any agency thereor. and opinions of authors expressed hercin do not necessarily state or thereof. The views United States Government or any agency thereof.

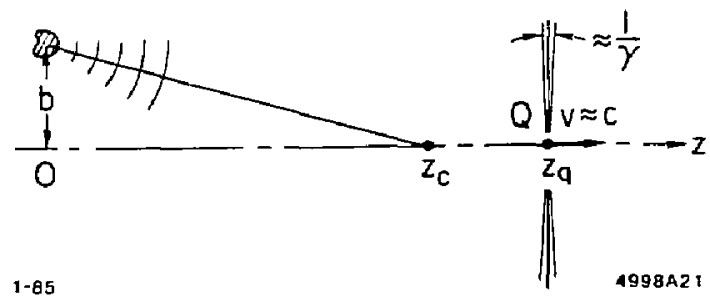

Figure 1. Figure showing the radiation field from a perturbing metallic object excited by the field of a relativistic charge moving along the $z$-axis. 


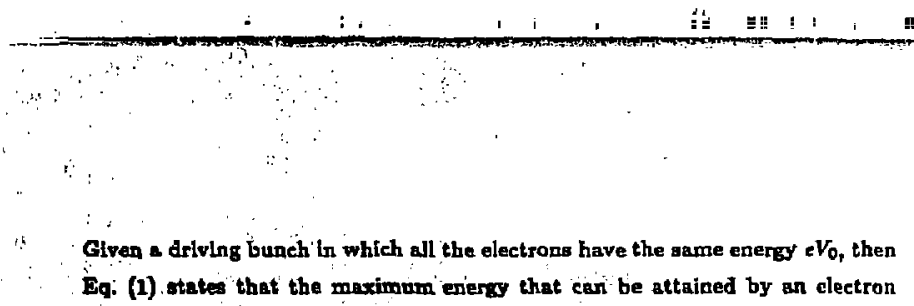
following behind the drlving bunch is $e R V_{0}$; assuming electrong in the driving i) binch which experience the moximum retarding feld are just brought to rest. Equation (2) is the efficiency for the tranglar of energy from the driving bunch to enerios u per unit length of structure in the wake fields. Equation (3) is a figure of merit for the conversion of field energy per unit length into accelerating gradient. The parameter $k_{i}$ scales inverselysat the square of the trangverse dimensions of is $=$ the strueture. In Eg. (4) $k_{n}$ is another fgure of merit giving the accelerating gradient produced per unit driving charge. Note that it is inconsistent to have

$\therefore$ both a high traniformer ratlo and a large $k_{a}$. A third loss parameter can also be defined: $k_{w} \equiv u / q^{2}$. The three loss parameters are related by $k_{a}^{2}=k_{2} k_{\mathrm{u}}$. For a .ingle if mode $k_{c}=k_{\mathrm{a}}=k_{\mathrm{w}}$, and all three loss parameters are identically equal to the if loss parameter as usually defined.

Let us next consider the wakè fields set up by some simple metallic boundary discontinuities having cylindrical symmetry. In Fig. 2(a) a charge moving in free space at $v$ a $c$ entere along the axis of a section of perfectly conducting pipe with infinitely thin walls. The field lines are not perturbed by the pipe in the limit $\gamma \rightarrow \infty$, either upon entering or leaving, and consequently no wake fields are produed. The gituation is different for the configuration shown in Fig. 2(b). Here it is asromed that the fields from the charge have been confined inside the pipe for $z<0$. As the charge exter from the plpe at $z=\%$, a toroidal radiation field is Get op as shown by the dashed lines. The aurface charge splita into two portions, a charge $Q_{1}$ on the bitside of the pipe and a charge $Q_{2}$ on the inside, with $Q_{1}+$ $Q_{2}$ equal to the driving charge $Q$. The initial ratio $Q_{2} / Q_{1}$ can be determined by minimtang the energy in the radiation field, ${ }^{2}$ giving in this case $Q_{1}=Q_{2}=Q / 2$. However, computer simulations on similar problems indicate that the radiation Gidd may not be confired to a delta-function wavefront expanding at the vejocity ef light, but rather might be smeared out over the region behind the wavefront $\because$ in a complicated way. In addition, the ratio $Q_{2} / Q_{1}$ does not seem to remain $r=:$

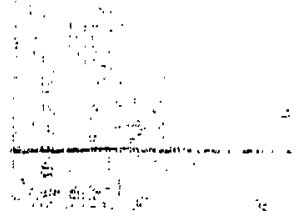

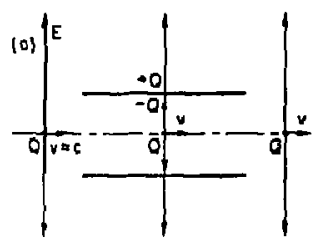

(b)

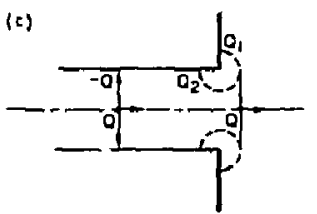

(d)
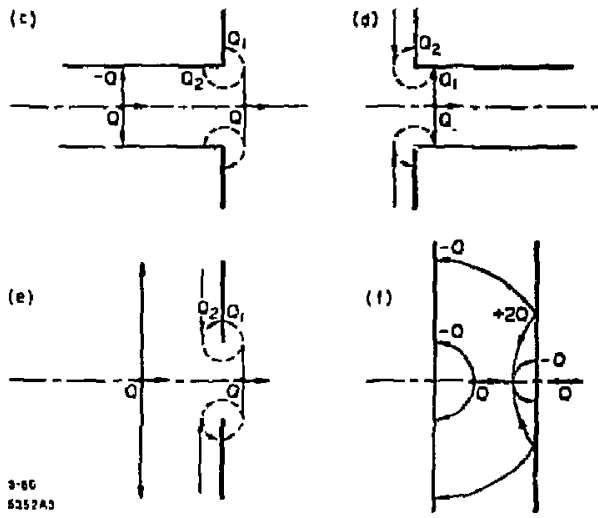

Figure 2. Fields excited by a point charge goving with $v \approx c$ past several cylindrically symmetric discontinuities.

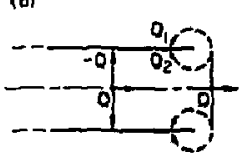

325209 
constant in time. Very probably, the total charge on the inside of the pipe decreates with increasing time. This geemingly simple boundary value problem hes not, however; been solved analytically.

Figures 2(c), 2(d) and 2(e) thow some additional elementary geometries, with their associated toroidal wake felds. Again, it should be emphasized that these apparesitly elementary boundary value problems have not been solved analytically. The details of the radiation field, indicated only schematically by the dashed lines, are in fact the subject of some controversy. Hand-waving argumentgingve been used to prove that the radiation field is, or is not, confined to a oharp wavefront. It has also been argued ${ }^{3}$ that in these three cases there are no wake fields at all in the region $r<a$, where $c$ is the radius of the eperture.

The situation is diferent for the case of Fig. 2(f), which shows a point charge passing between two parallel metallic plates. This case is important in that two parallel planes give the same wake fields as a pillbox cavity for dialances behind the driving cbarge such that $s<$ so where

$$
s_{0}=\left(g^{2}+4 b^{2}\right)^{1 / 2}-g
$$

Here $b$ is the cavity radius and $g$ the gap spacing between the feces of the pillbox. In other words, if $s<$ so the signal induced by a driving charge as it entera through the first face of the pillbox can propagate to the outer cylindrical wall, be reflected, and return to the axis to influence a trailing test charge before the test charge exito through the second face of the pillbox. The pillbox cavity is important becanse it can often serve as a primitive model for an accelerating structure. The wake felds for the case of Fig. 2 (f) have been calculated analytically (see, for example, Ref. 4).

Of course, the detailed behavior of the wake felds in time and space is not the feature of main interest. What we really want to know is the net energy gain or the net transverse kick integrated through a structure for a test particle following at a fixed distance behind the driving charge, assuming that both the driving charge and the test particle are traveling at $v=c$. The Jopgitudinal wate potential per unit driving charge is then defined by

$$
\left.W_{x}(s)=-\frac{1}{q} \int_{s_{i}}^{s} d z \mid E_{x}(z, t)\right]_{t=(z+r) / \mathrm{c}}
$$

assuming both driving and test charges are traveling parallel to the $z$ axis. Here $z_{2}-z_{1}$ is the region over which the wake fields are appreciable for $\mathrm{a}$ finite structure, or the periodic length for a periodic structurc. A similar expression holds for the transverse wake potential:

$$
W_{\perp}(s)=\frac{1}{q} \int_{x_{1}}^{x_{1}} d z\left[\mathrm{E}_{\perp}+(v \times \mathrm{B})_{\perp}\right]_{q=(x+s) / c}
$$

In the above expressions the position of the driving charge is assumed ta be $z=c t$, and the wake fields are integrated in a frame of reference moving with the test particle.

As mentioned above, analytic expregsions have been obtained for the wake fields (the transition radiation) generated by a point charge entering and leaving the region between two parallel metallic planes. Using the $E_{x}$ field compopent derived in Ref. 4, K. Bane ${ }^{5}$ has carried out the integration in Eq. (5a) to obtain the delta function wake potential for this case:

$$
\begin{aligned}
& 2 \pi \epsilon_{0} W_{z}(s)=2 \delta(s) \ln \left[\frac{g}{s}\right]-2 \sum_{n=1}^{\infty} \delta(2 n g-s) \ln \left[\frac{s^{2}}{(s+g)(s-g)}\right]
\end{aligned}
$$

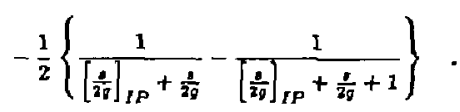

Here $g$ is the separation between the planes and $I P$ meass the integer part of the term in brackets. This function is shown in Fig. 3. Note that the wake 


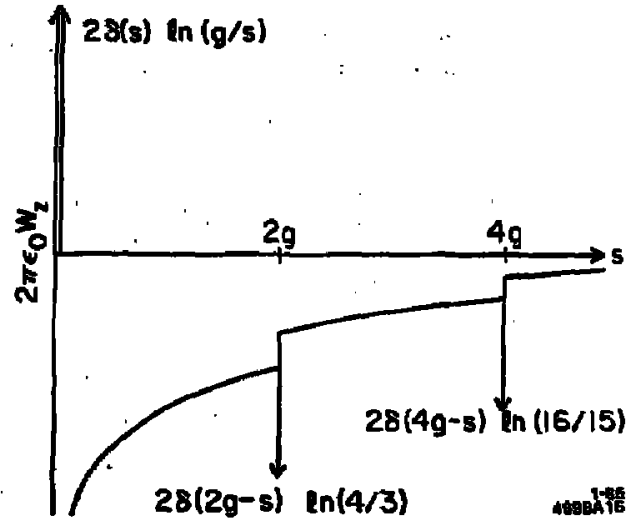

Figure 3. Longitudinal delte-function wake potential for a point charge moving perpendicular to two parallel metalic planes. is accelerating $\left(W_{s}<0\right)$ for all $s>0$. However, the wales sean by the drivine charge itself at $B=0$ is rotarding, and the transtormer rablo an defined proviously is always less than one.

A simillar situation in encountered in the case of a point driving charge moving at $v \approx e$ on the axis of a hollow tube with resiative walle. Again, the wake is accelerating behind the driving charge, exceph for a very amall reglon within a critical distance (.01 mm for a point charge moving in copper tube $8 \mathrm{~cm}$ in radius) immediately bahind the changer It can be ahown that for wuch a resistive wall tube the transformer ratio is less than $1 / \sqrt{\pi}$ (see Ref. 6, Sec. 3.2).

Once the wate potential for a unit point charge is known, the potential at any point within or behind an arbitrary charge distefbution with llne donsity $\rho[0]$ can be computed by

$$
V(s)=-\int_{4}^{\infty} W_{r}\left(s^{\prime}-s\right) \rho\left(d^{\prime}\right) d s^{\prime} .
$$

The total loss paramotar $k_{4}$ is then obtained from

$$
k_{u}=\frac{1}{q^{2}} \int_{-\infty}^{\infty} V(s) \rho(s) d s .
$$




\section{Wake Potentials for Closed Cavities and Periodic Structures}

Consider a driving charge $Q$ moving at velocity $v$ through a closed cavity with perfectly conducting walls, as ghown in Fig. 4. A test charge also moves at the same velocity, but on a path which parallel to that of the driving charge and at a longitudinal distances behind. Our goal is to calculate the longitudinal and transwerse wike potentials experiened by the test charge in the limit $v \approx c$. For $v \neq c$ the expressions for the wake potentials are in general mueh more complicated and the wake potential concept is less useful.

Under certain rather general conditions, which will be spelled out in detail later, it can be shown ${ }^{6,7}$ that the longitudinal and transverse wakt potentials can be written in terms of the properties of the normal modes of the charge-frec cavity in a relatively simple way:

$$
\begin{aligned}
& W_{z}\left(r^{\prime}, r_{1} s\right)=2 H(s) \sum_{n} k_{n}\left(r^{\prime}, r\right) \cos \frac{\omega_{n} s}{c} \\
& W_{\perp}\left(r^{\prime}, r, s\right)=2 H(s) \sum_{n} k_{n \perp}\left(r^{\prime}, r\right) \sin \frac{\omega_{n} s}{c}
\end{aligned}
$$

where

$$
H(s) \equiv \begin{cases}0 & s<0 \\ 1 / 2 & s=0 \\ 1 & s>0\end{cases}
$$

and

$$
\begin{aligned}
k_{n}\left(r^{\prime}, \mathbf{r}\right) & =\frac{V_{n}^{\prime}\left(\mathbf{r}^{\prime}\right) V_{\mathrm{n}}(\mathbf{r})}{4 U_{\pi}} \\
k_{\mathrm{n} \perp}\left(\mathbf{r}^{\prime}, \mathbf{r}\right) & =\frac{V_{n}^{(}\left(\mathbf{r}^{\prime}\right) \nabla_{\perp} V_{n}(\mathbf{r})}{4 U_{n} \omega_{n} / c} .
\end{aligned}
$$

Here $w_{n}$ is the angular frequency the $n$th mode, and $V_{n}(r)$ is the vollage that would be gained by a nonperturblng test particle crossing the cavity in which

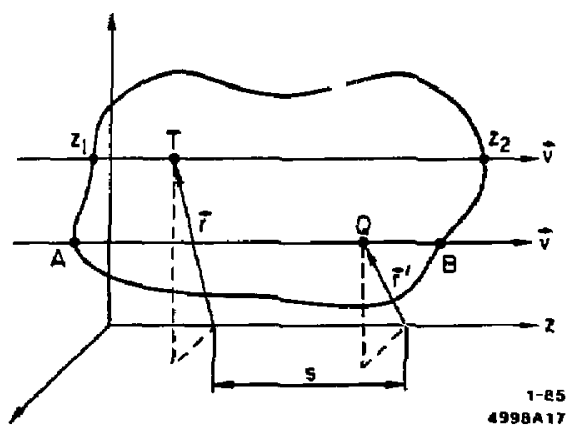

Figure 4. 1 driving charge $Q$, moving at constant velocity $v$ parallel to the $z-$ axis, enters a elosed cavity at $A\left(r^{\prime}, z=0\right)$ at $t=0$ and leaves at $B\left(r^{\prime}, z=L\right)$. $A$ non-perturbing test particle $T$ also mo"os at the same velocity $v$, but at transverse position $r$ and at longitudinal distance $s$ behind $Q$. 
energy $U_{n}$ is stored in the nth mode. Assuming the clectric field for the $n^{\text {th }}$ mode varies with time as exp(iwt) and the position of the test particle is given by $z=e t$, this voltage is

$$
V_{n}(r)=\int_{s_{1}}^{\eta} d z E_{x}(r, z) \exp \left(\frac{i \omega_{n} z}{c}\right)
$$

The conditions under which Eqs. (9) are valid for the longitudinal and transverse wake functions are discussed in detail in Refs, 6 and 7 , and are summarized in Table I. We see that if the driving charge and test particle follow different pathe in a closed cavity of arbitrary thepe, neither Eq. (9a) nor ( $9 b$ ) give a valid description of the wake potentials. If the particles follow the same path in a closed cavity of arbitrary shepe, Eq- (9a) is valid for the longitudinal wake potential but Eq. (9b) does not correctly describe the transverse wake patential. Formal expressions can indeed be written down for the non-valid cases, but the integrals are much more complicated, and the wake potentials for a given mode do not separate neatly into a product of an s-dependent factor and a factor which depends only on $r$.

Note that Eqs. (9a) and (9b) are related by

$$
\frac{\partial W_{\perp}}{\partial s}=\nabla_{\perp} W_{s}
$$

This relation between the longitudinal and transverse wakes is sometimes termed the Panofsky-Wenzel theorem. ${ }^{8}$ It was originally derived to calculate the transverse momentun kick seceived by a nonperturbating charge traversing a cavity excited in a suggle if mode.

The wake potential formalism, using properties of the charge-free cavity modes, makes it pessible to calculate useful quantities for the charge-driven cavity. An important example is the longitudinal wake potential for the case in
Table I

\begin{tabular}{|c|c|c|c|}
\hline & Cuse & $\begin{array}{c}\text { Eq. (9a) Valid } \\
\text { for } W_{x}\end{array}$ & $\begin{array}{l}\text { Eq. (9b) Valid } \\
\text { for } w_{\perp}\end{array}$ \\
\hline (a) & $\begin{array}{l}\text { Test charge and driving cinarge } \\
\text { follow different paths in a closed } \\
\text { cavity of arbitrary shape. }\end{array}$ & No & No \\
\hline (b) & $\begin{array}{l}\text { Test charge and driving charge } \\
\text { follow the same path in cavity of } \\
\text { atbitrary shape. }\end{array}$ & Yes & No \\
\hline (c) & $\begin{array}{l}\text { Velocity } v \text { is in the direction } \\
\text { of aymmotsy of a right cylinder } \\
\text { of arbitrary cross section. }\end{array}$ & Yos & Yes \\
\hline (d) & $\begin{array}{l}\text { Both driving charge and test } \\
\text { charge move in the beam tube } \\
\text { region of an infinite repeating } \\
\text { structure of arbitrary cross section. }\end{array}$ & Yes & Yes \\
\hline (e) & $\begin{array}{l}\text { Both particles move near the } \\
\text { exis of any cylindrically } \\
\text { symmetric cavity. }\end{array}$ & Yes & Yes \\
\hline
\end{tabular}

Cases for which Eqs. (9a) and (9b) give the wake potentials in the limit $v \approx c$

which the test charge and driving charge follow the same path. Equations (9a) and (10a) reduce to

$$
\begin{gathered}
W_{*}(r, s)=\sum_{n} k_{n}(r) \cos \frac{\omega_{n} s}{c} \times \begin{cases}0 & s<0 \\
1 & s=0 \\
2 & s>0\end{cases} \\
k_{n}(r)=\frac{\left|V_{n}(r)\right|^{2}}{4 U_{n}} .
\end{gathered}
$$

The potential seen by the charge itself is

$$
V(r, 0)=-q W_{z}(r, 0)=-q \sum_{n} k_{n}[r]
$$




$$
V_{n}(\mathbf{r}, 0) \equiv V_{s}(0)=-q k_{m}
$$

The exierts left behind in the $n^{\text {th }}$ moda after the driving charge has left the cavity

$$
U_{n}=-q V_{n}(0)=q^{2} k_{n}
$$

The parameler of is the constant of proportionality between the energy lost to the ath mode and the aquare of the driving charge, hence the name loss parameter or lows finctor.

Nole from $\mathrm{Eq}$. (13) that an infinitesinal distance behind a driving point charge the potential ia reterding for the $\mathrm{gth}$ mode with magnitude

$$
V_{n}\left(0^{+}\right)=2 V_{n}(0)=-2 q k_{n}
$$

As a funetion of distance $s$ behind the driving charge, the potential varies as ?

in:

1

$$
V_{n}(s)=V_{n}\left(0^{+}\right) \cos \frac{\omega_{n} s}{c}=-2 g k_{n} \cos \frac{\omega_{n} a}{c} .
$$

Dquition (166) expreses what is somntimes termed the fundamental theorem of benm loading": the voltage induced in normal mode by a foint charge is exactly twice the ratiraing voltige een by the charge itselr. The transformer ratio for a point charge exciting a single mode is then also exactly equal to two. It is readily chown that thiti factor of two follows directly from conservation of energy. In See \& we will bhos that this restrietion on the transformer ratio for a point charge doẹ not necessarily apply to an extended driving charge distribution.

A physlcal wale for a real cavity is a summation nver many modes. Perhaps the modes might add up to produce a transformer ratio greater than two, even for a polat eharge We note, however, that the wake for each mode varies with is $W_{n}=2 k_{n} \cos \left(\omega_{n} s / c\right)$. At $a=D^{+}$the wakes all add in phase, and the sum of the whes for all the modes gives a retarding potential which is exactly twice the retarding potential acen by the driving eharge itself at $\theta=0$. At any value of $s$ where the net wake is accelerating, the cosino wakes for the indivicual modes can never do better than add exactly together in phase, as they do at $a=0^{+}$. Thus

$$
|W(s)| \leq \sum_{n} W_{n}\left(s=0^{+}\right)=2 \sum_{n} W_{n}(s=0)
$$

and the transformer ratio for a real cavity with many modes, driven by a point charge, is equal to or less than two. In practice it will be considerably leas than two, since the modes will never come close to adding in phase anywhere oxcept at, $s=0^{+}$.

It is easy to show that Eq. (17) also follows from consarvation of onorgy. Consider a point driving charge qI which loses energy

$$
U_{1}=q_{1}^{2} W(0)
$$

to the wake fields in a cavity (remember that $W(0)$ is alsways positive by dofnition). The arcolerating potential at position $s$ is $-q_{1} W(s)$. By superposition the energy gained by a charge $\eta_{z}$ at position $s$ is

$$
U_{2}=-q_{2}\left|q_{1} W(s)+q_{2} W(0)\right|
$$

Assume first that $q_{1}$ and $q_{2}$ have the same sign. by conservation of onaray $U_{2} \leq U_{1}$ and uaing $E_{q s},(18 a)$ and (18b) the transiormer ratio $R=-W(\mathrm{~d}) / W(0)$ is

$$
R \leq \frac{q_{1}^{2}+q_{2}^{2}}{q_{1} q_{2}}=\frac{1+\alpha^{2}}{\alpha}
$$

where $\alpha \equiv q_{2} / q_{1}$ is positive. This inequality mist hold for any value of $\alpha_{1}$ in particular for $\alpha=1$ which minimizes the right-hand side, leading to $R \leq 2$. If $q$ and $q_{2}$ are opposite in sign, the transiormer ralio is redefined as $R^{\prime}=-\boldsymbol{R}_{1} a$ is replaced by $-|\alpha|$, and the above expression ngain gives $R^{\prime} \leq 2$, 
If the driving charge and the accelerated charge follow different paths threugh the cavity, the altuatios becomes more complieated, We figt note from Exs. (9) and (10) that the loingitudinal wake potential is unchanged if the paths of the driving charge and the test charge are interchanged. This oymmetry with respect to the intercbange of $\mathrm{r}$ and $\mathbf{r}$ bs alas an alternative expression of the Lorents reciprocity theoren, derived in standard texts on microwave theory. ${ }^{10}$ If we now apply conservation of energs to two charges $q$ and $q 2$ following different paths, we can show that

$$
\left|W_{12}(s)\right|=\left|W_{21}(s)\right| \leq 2\left[\left.W_{1}(0) W_{2}(0)\right|^{1 / 2},\right.
$$

where $W_{12}(s)$ is the wake along path 2 produced by a charge travelling on path 1 , and so forth. If we define a transformer ratio $R_{12}$ by

$$
R_{12}(s) \equiv \frac{\left|W_{12}(s)\right|}{W_{1}(0)}
$$

and aimilarly for $R_{31}$, than for any value of

$$
\begin{aligned}
& R_{12} \leq 2\left[\frac{W_{2}(0)}{W_{1}(0)}\right]^{1 / 2}=2\left[\frac{\sum_{n} k_{n}\left(r_{2}\right)}{\sum_{n} k_{n}\left(r_{1}\right)}\right]^{1 / 2} \\
& R_{n 1} \leq 2\left[\frac{W_{1}(0)}{W_{2}(0)}\right]^{1 / 2}=2\left[\frac{\sum k_{n}\left(r_{1}\right)}{\sum_{n} k_{n}\left(r_{2}\right)}\right]^{1 / 2}
\end{aligned}
$$

and

$$
R_{12} R_{21} \leq 4
$$

The case of a periodically repeating structure is of obvious importance in accelerator deaign. Although real periodic structures are of conrse never infinite, practical structurea al least a few periods in length seem to fulfill condition (d) of
Table I. Thus the wake potentials can be computed by a summration over normal modes. For the case of a cylindrically aymmetric structure, all modes depend on the aximuthal angle $\phi$ as $e^{i m \phi}$. The wake potentials can then be written for $a>0$,

$$
\begin{aligned}
W_{a m}= & 2\left(\frac{I^{\prime}}{a}\right)^{m}\left(\frac{r}{a}\right)^{m} \cos m \phi \sum_{n} k_{m h}^{[a)} \cos \frac{\omega_{m n} s}{c} \\
W_{\perp m}= & 2 m\left(\frac{r}{a}\right)^{m}\left(\frac{r}{a}\right)^{m-1}(r \cos m \phi-\dot{\phi} \sin m \phi) \\
& \times \sum_{n} \frac{k_{m h}^{(a)}}{\omega_{m n} a / c} \sin \frac{\omega_{m n} s}{c} .
\end{aligned}
$$

Here $\hat{f}$ and $\hat{\phi}$ are unit vectors and $k$ (s) is the loss factor per innit length calculated at $\mathrm{r}=a$, where $a$ is the radius of the beam tube region. That is

$$
k_{n}^{(a)} \equiv \frac{\left[E_{s n}(r=a)\right]^{2}}{4 u_{n}}
$$

where $u_{n}$ is the energy per trit length in the $n^{\text {th }}$ mode. The longitudinal cosinelike wake potential per period for the SLAC strueture is shown in Fig. 5. Note the very rapid fall-ot in the wake immediately behind the driving charge, from a peak wake of $\mathrm{B} \mathrm{V} / \mathrm{PC}$ per period at time $t=s / c=0^{+}$. The wake seen by a point charge would be just one half of this wake, of $4 \mathrm{~V} / \mathrm{pC}$. The sine-like tranverse dipole $(m=1)$ wake potentin) for the SLAC structure is shown in Fig. 6. This figure illustrated the fact thet the total wake potential is obtained by aumming a finite number of modes that can be obtained using a reasonable computation time, and then adding an a so-called analytic extension to take into account the contribution from very high frequency modes. Details are discussed in Ref. 11.

If the dimensions of a particular otructure are scaled by a factor $F$, the trequencies of the normal modes scale as $F^{-1}$. The amplitude of the longitudinal wake potential per unit length of strueture seales as $F^{-2}$ at time $F t$, or in terms 


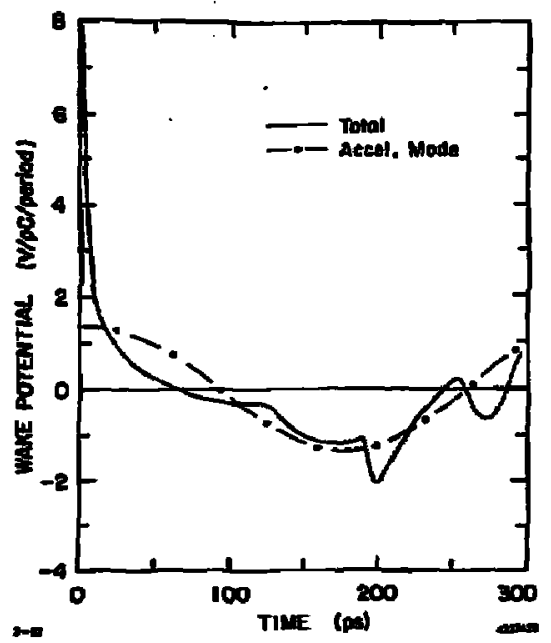

Tigure 5. Iongitudinal delta-function wale potential per cell for the BLAC

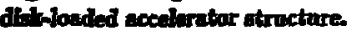

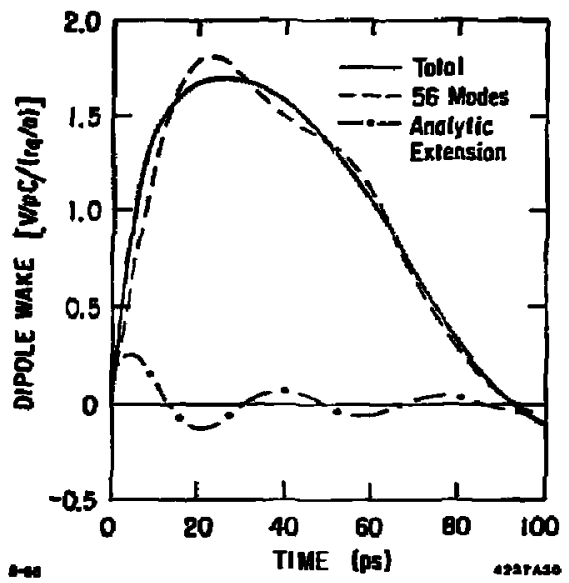

Figure 6. Dipole delta-function wake patential per cell for the SLAC structure. 
of the modal fraquencies wa $\omega_{n}^{2}$. The an ilitude of the dipole walke polential per unit length at time $F t$ seales as $F^{-1}$, or es $\omega_{n}^{3}$.

\section{Wake Potentials on a Collinear Path with a Charge Distribution}

- in the lastgection, the wake potentials due to a point driving charge traversing a clased covity were considered under rather general conditions. In this section wo confine our attention to the case in which the driving charge and test particle follow the oame poth thropgh a cavity or strueture, but we allow the driving charge to ba a distributios auch that the line densily is given by $\rho(s)=I(t) / \mathrm{c}$. The potential at tlmet $=0 / \mathrm{e}$ is then

$$
V(t)=-\int_{-\infty}^{t} I\left(t^{\prime}\right) W_{4}\left(t-t^{\prime}\right) d t^{\prime}
$$

For a point charge wa found previously that

$$
V(t)=-2 q \sum_{n} k_{n} \cos \omega_{n} t
$$

If such o eharge hoving initial energy $q V_{0}$ is just broughe to rest by the retarding wake potential at $t=0$, then $V_{0}=q \sum_{n} k_{n}$ and

$$
V(t)=\frac{2 V_{0} \sum_{n} k_{n} \cos \omega_{n} t}{\sum_{n} k_{n}}
$$

If the atructure supports only a single mode, then $V(t)=-2 V_{0} \cos \omega_{n} t$. However, a jhyited bunch, even a very short bumch, consists of a large number of individual - charged which are not rigidly connected. Thus the leading charge in such a physically real bunch will experience go deceleration, while the trailing charge will expariance the full induced voltage, or tivice the average retarding valtage per particle (assuming the bunch length is short compared to the wavelengths of all modes with appreciable values of $k_{m}$ ). The wake potential for a short charge distribution extending from $t=0$ to $t=T$, interacting with a single mode, is illugtrated in Fig- 7(a). Within the bunch the potential is given by

$$
V(t)=-\frac{2 V_{0}}{4} \int_{0}^{t} I\left(t^{\prime}\right) d t^{\prime}
$$

where $V_{0}$ is the avarage energy lose per particle in the distribution. Thb cen be seen by substituting Eq. (24) in

$$
V_{0}=\bar{V}(t)=\frac{1}{q} \int_{0}^{T} V(t) I(t) d t
$$

and working out the double integral. Note from Eg. (24) that for $t=T$ at the end of the distribution $V(T)=-2 V_{0}$. Therefore $V_{m}^{+}=2 V_{0}, V_{m}=\left|-2 V_{0}\right|=2 V_{0}$ and the transiormer ratio is $R=V_{m}^{+} / V_{m}=1$.

The potential in and behind a long charge distribution is shown schematically in Fig. $T(b)$. We consider first the case for a single mode. From Eq. (22) with $W_{\varepsilon}(t)=2 k_{n} \cos \omega_{n} t_{1}$

$$
V_{n}(t)=-2 k_{n} \int_{-\infty}^{t} I\left(t^{\prime}\right] \cos \omega_{n}\left(t-t^{\prime}\right) d t^{\prime}
$$

Assume now that the bunch extends in time from $-T$ to $+T$. Withln the bunch $(-T<t<T)$ the retarding potential is

$$
V_{n}^{-}(t)=-2 k_{n}\left[\cos \omega_{n} \int_{-T}^{t} I\left(t^{\prime}\right) \cos \omega_{n} t^{\prime} d t^{\prime}+\sin \omega_{n} t \int_{-T}^{t} I\left(t^{\prime}\right) \sin \omega_{n} t^{\prime} d t^{\prime}\right] \cdot(26)
$$




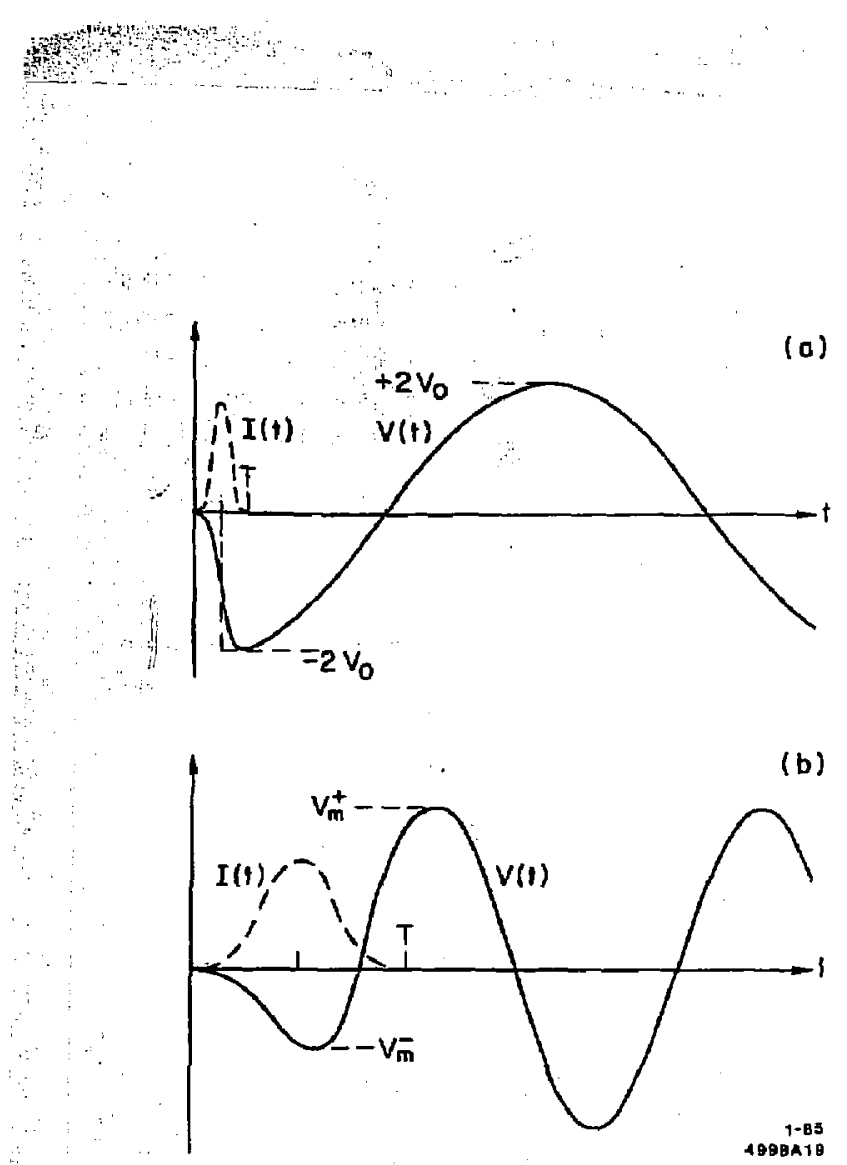

Figure 7. Potential in and behind a charge distribution interacting with a single mode for (a) a short bench, and (b) a long buncb.
Following the bunch $(t>T)$ the accolerating potential is

$$
V_{n}^{+}(t)=2 k_{n}\left[\cos \omega_{n} t \int_{-T}^{T} I\left(t^{\prime}\right) \cos \omega_{n} t^{\prime} d t^{\prime}+\sin \omega_{n} t \int_{-T}^{T} I\left(t^{\prime}\right) \sin \omega_{n} t^{\prime} d t^{\prime}\right] .
$$

If the busch is symmetric about $t=0$, the second integral in Eq. (27) vanishes, and $\boldsymbol{V}^{+}(t)$ reachea a maximum value given by

$$
V_{m}^{+}=2 k_{n} \int_{-T}^{T} I\left(\iota^{\prime}\right) \cos \omega_{n} t^{\prime} d t^{\prime}
$$

'The retarding potential at the center of such a symmetric bunch is given by

$$
V^{-}(0)=-2 k_{\mathrm{n}} \int_{-T}^{0} I\left(t^{\prime}\right) \cos \omega_{\mathrm{n}} t^{\prime} d t^{\prime}=-\frac{1}{2} V_{m}^{+}
$$

If $V-(0)$ happens also to be the maximum (absolute) value of the retarding potential, then $\left|V^{-}(0)\right|=V_{m}^{-}$, and the transtormer ratio is $R=V_{m}^{+} / V_{m}^{-}=2$. If $V^{-}(0)$ is not at the peak of the retarding potential, then $V_{m}^{-}>\left|V^{-}(0)\right|$ and $\boldsymbol{R}<2$. Thus for symmetric bunches interacting with a single mode, the transformer ratio cannot exceed two. This upper limit is reached only if the maximum retarding potential is reached at the center of symmetry of the distribution. Otherwise, the transformer ratio is jess than two. If the bunch is not symmetric, the preceding argument does rot apply. The transformer ratio can then in prineiple be arbitrarily large, as we will see shortly.

Even for symmetric bunches in a physical structure, which has many modes, the limitation $R \leq \mathbf{2}$ tends to apply. For example, Fig. $B$ shows potentials for a Gaussian distribution interacting with the SLAC accelerating structure for several valuss of bunch length. Note that, for a bunch length such that the peak retarding potential is reached near the center of symmetry of the bunch 


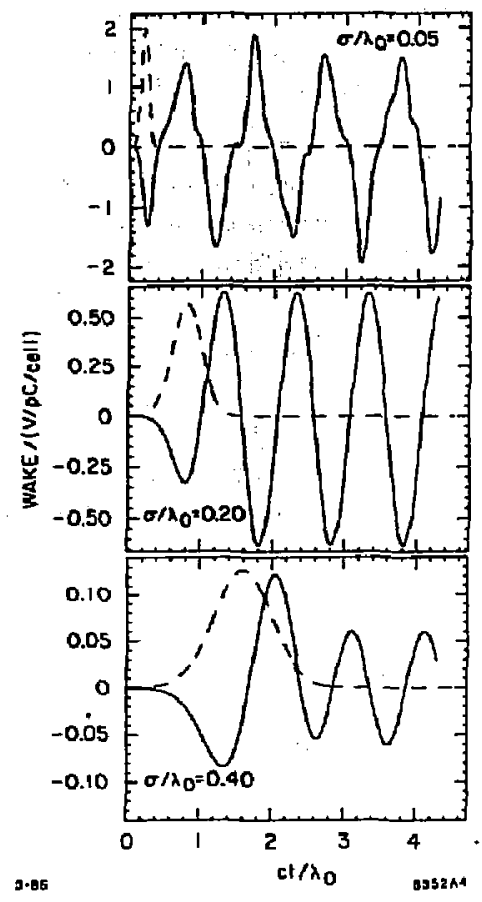

Figure 8. Potential in and behind a gaussian bunch interacting with the lonbitudinal modes of the SLAC structure. The transformer ratio for $\sigma / \lambda_{0}=0.05$, 0.20 and 0.40 are $1.4,1.9$ and 1.4 reapectively. $\left(\sigma / \lambda_{0}=0.2\right)$, the transformer ratio is approximately equal to two, while for both longer and shorter bunch lengths the transtormer ratio is considerably iess than two. It can be shown ${ }^{9}$ that for a Gaussian bunch interacting with a single mode the loss parameters $k_{n} \equiv u / q^{2}$ and $k_{a} \equiv E_{a} / 2 q$ are related to the loss parameter $k_{\ell} \equiv E_{a}^{2} / 4 u$ by

$$
\begin{aligned}
& k_{\mathrm{u}}=k_{L} e^{-\omega_{n}^{2} \sigma^{2} / c^{2}}=k_{L} e^{-4 \pi^{2} \sigma^{2} / \lambda_{n}^{2}} \\
& k_{\mathrm{a}}=k_{\ell} e^{-\omega_{n}^{2} \sigma^{2} / 2 c^{2}}=k_{l} e^{-2 \pi^{2} \sigma^{2} / \lambda_{n}^{x}}
\end{aligned}
$$

for each mode. Thus as the bunch length increases, coupling to higher modes is rapidty suppressed by the exponential factor. For the SLAC atructire, $k_{\ell} p=$ $0.70 \mathrm{~V} / \mathrm{pC} / \mathrm{cell}$ for the fundamental mode, where $\mathrm{p}$ is the cell length. The amplitude of the accelerating mode voltage per cell excited by a Gaussian bunch with total charge $q$ is therefore

$$
\frac{V_{L}}{q}=2 k_{a} p=1.40 e^{-2 \pi^{2} \sigma^{2} / \lambda_{a}^{2}} \quad \mathrm{~V} / \mathrm{pC} / \text { cell }
$$

For $0 / \lambda_{0}=0.05,0.20$ and 0.40 , this gives $\nu_{1} / q=1.33,0.64$ and $0.08 \mathrm{~V} / \mathrm{pC} / \mathrm{cel}$. These values agree well with the computer calculation shown in Fig. 8.

The plot for $\sigma / \lambda_{0}=0.4$ in Fig. 8 also illustrates the phenomenos of autoacceleration, in which fields induced by particles at the front of the bunch can accelerate particles at the tail of the same bunch.

It is possible in principle to design a structure in which the accelerating potentials for several modes superimpose maximally at some point behind the bunch to produce $R>2$. Consider, for example, a two-mode structure with loss factors $k_{0}$ and $k_{1}$ and frequencies $\omega_{0}$ and $\omega_{1}$ related by $\omega_{1}=3 \omega_{0}+\delta$, where $\delta / \omega_{0}$ is a small quantity (if $\delta=0$ the maxima of the wakes for the two modes would never superimpose). Assume a rectangular bunch extending from $-T$ to $T$ with constant current $J(t)=I$. From Eq. (22), using also $W_{s}(t)=2 k_{n} \cos \omega_{n} t$, the 


\section{retarding patentlal within the bunch is}

$$
V-(t)=-2 I\left[\frac{k_{0}}{\omega_{0}} \sin \omega_{0}(t+T)+\frac{k_{1}}{\omega_{1}} \sin \omega_{1}(t+T)\right]
$$

Behind the buinch $(t>M)$,

$\because \because$

$$
V+(t)=-4 I\left[\frac{\omega_{0}}{\omega_{0}} \cos \omega_{0} t \sin \omega_{0} T+\frac{k_{1}}{\omega_{1}} \cos \omega_{1} t \sin \omega_{1} T\right]
$$

If we choose $\omega_{0} T=\pi / 2$ and $k_{1}=k_{0}$, we find the minimum potential inside the bunch and the maximum potential behind the bunch are

$$
V_{m}^{-} \approx \frac{8}{3 \sqrt{2}} \frac{k_{0} I}{w_{0}} \text { and } V_{m}^{+}=\frac{16}{3} \frac{k_{0} I}{w_{0}}
$$

\section{and therefore $R \approx 2 \sqrt{2}$}

This calculation can be generalized to atructures with many modes related by $\omega_{n}=(2 n+1) \omega_{0}+\delta_{n}$. If the lose factors are equal for all of the modes, it is itraightforward to ohow

$$
R \propto\left(\frac{8}{x}\right)\left(1+\frac{1}{3}+\frac{1}{5}+\ldots\right)
$$

It can be argued that auch a atructure is unphysical. On the other hand, there is no reaion to believe that the two mode atructure described above is not realinable. The $\sqrt{2}$ gain in tranaformar ratlo over the aingle mods case in, however, quite riodent.

Iet us now retum to the case of an asymmetric driving bunch. Take as an example a triangular current raup in a single mode cavity. Let $I(t)=$ Iwt for $0<t<T$ and $I(t)=0$ othenviace. For simplicity let the bunch length be
$T=2 \pi N / \omega$, where $N$ is an intager. Then within the bunch

$$
V^{-}(t)=2 k I \omega \int_{D}^{t} t^{\prime} \cos \omega\left(t-t^{\prime}\right) d t^{\prime}=-\frac{2 k I}{\omega}(1-\cos \omega t)
$$

whereas behind the bunch

$$
V^{+}(t)=2 k \pi \omega \int_{0}^{T} t^{\prime} \cos \omega\left(t-t^{\prime}\right) d t^{\prime}=2 k I T \sin \omega t .
$$

Thus $V_{m}^{-}=4 k J / \omega, V_{m}^{+}=2 k J I=4 \pi k I N / \omega$ and

$$
R=\frac{V_{m}^{+}}{V_{m}^{-}}=\pi N \quad\left\{\begin{array}{c}
\text { current ramp, } \\
\text { single mode }
\end{array}\right\} .
$$

The wake potentials for a current ramp of length $N=2$ interacting with a single made are shown in Fig, $9(\mathbf{a})$.

In a real structure with many modes, one might expect that the transformer ratio will be less than that given by $\mathbb{I} q$. (34). The potential axcitad in the SLAC gtructure by a current ramp with $N=2$ is shown ln Fig. 10. Within the bunch the retarding potential has a behavior closs to the aingle mode caleqlation, $V^{-}(t) \sim 1-\cos \omega t$. However, sorne erergy goes into higher modes, as lo evident by ripples on the cosine wave beinind the bunch. This causes a degradation of the transformer ratio from the gingle mode prediction $R=2 \pi$ to $R=4.86$. The degradation worsens as the bunch gets longer, as can be seen in FIg. 11 .

The efficiency for energy extraction from a driving bunch extenting from $t=0$ to $t=T$ in which all of the electrons have the bame enercy $e T_{0}=e V_{m}^{-}$is

$$
\eta=-\frac{1}{q V_{m}} \int_{0}^{T} I(t) V^{-}(t) d t .
$$

For a lincar current ramp interacting with a single mode, ovbstitution of Fo. (33a) together with appropriate expresigions for $I(t), V_{m}$ and $q$ into $E_{q}$. (36) gives an 


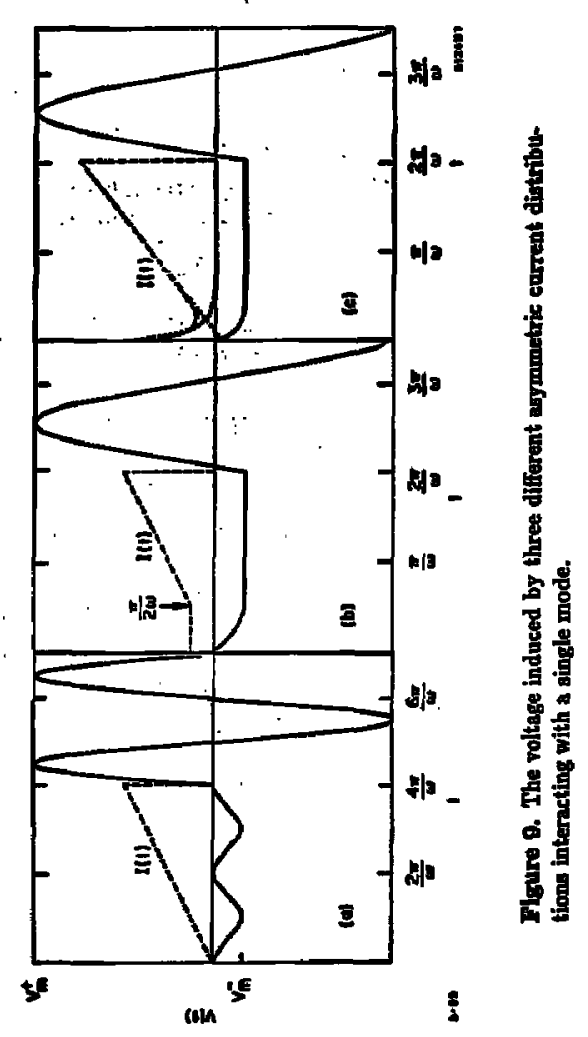

29

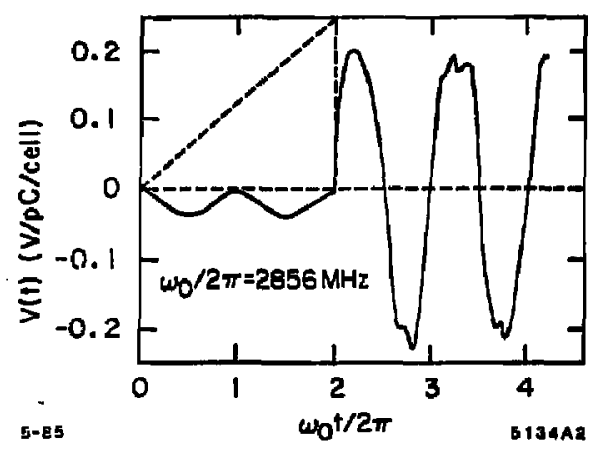

Figure 10. The potential induced by a linear current ramp interacting with the modes in the SLAC structure.

30 


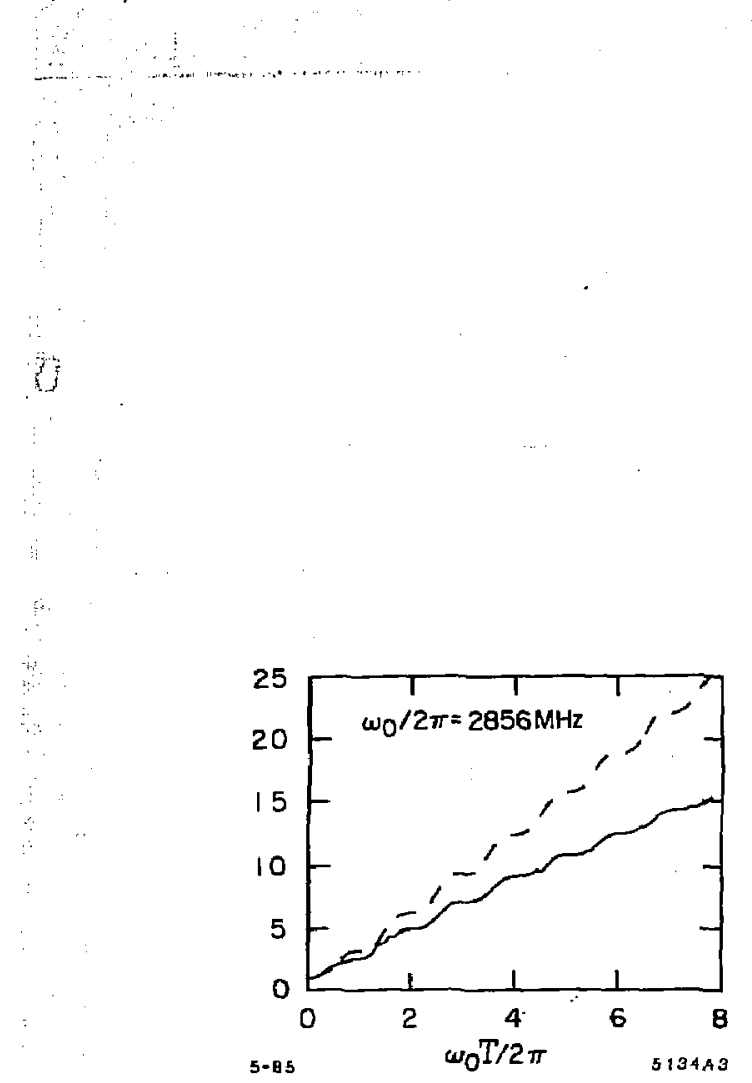

Figure 11. The transformer ratio for a linear current ramp in the SLAC structure as a function of bunch length. The dashed line gives single mode results. efficiency of 0.5 if $\omega T=2 \pi N . \Lambda$ higher efficiency and $z$ higher transformer ratio could be obtained if the retarding potential could be made as fat as possible across the current distribution. In the limit $V^{-}(t)=V_{m}^{-}=$constant, Eq. (35) gives an efficiency of 100\%. In Ref. 12 it is proven that the potential can be exactly flat only for a current distribution which consists of a delta function followed by a linear current ramp, where the proper relation exists between the value of the delta function and the slope of the current ramp. In this linit the transformer ratio is given by ${ }^{12}$

$$
R=\left[1+(2 \pi N)^{2}\right]^{1 / 2} \quad\left\{\begin{array}{c}
\text { Delta function plus } \\
\text { current ramp, } \\
\text { single made }
\end{array}\right\} .
$$

Here $N=\omega T / 2 \pi=c T / \lambda$, and $N$ can now take non-integer values. For lasge $N$ the transformer ratio approachcs $R \approx 2 \pi N$ and the efficiency approaches $100 \%$. The transformer ratio for the delta function alone $(N \rightarrow 0)$ is $R=1$, as we know is the tase for all ahort bunches, and the efficiency is 0.5. An approximation to this distribution, in which the wake potential is driven negative by an expontentially decaying spike and then hald constant, by a rising current ramp, is illustrated in Fig. g(c).

A third distribution of interest is a linear current ramp preceded by a quarter wavelength rectangular pulse. The response to this distribution is shown in Fig. 9 (b). The transformer ratio in the case of this "doorstep" distribution is ${ }^{12}$

$$
R=\left[1+\left(1-\frac{\pi}{2}+2 \pi N\right)^{2}\right]^{1 / 2} \quad\left\{\begin{array}{c}
\text { Doorstep plus } \\
\text { current ramp, } \\
\text { single mode }
\end{array}\right\} \text {. }
$$

In the limit of large $N$ the transformer ratio agair approaches $R \approx 2 \pi N$. For long bunches the transformer ratio and the efficiency are again approximately twice that for the linear current ramp alone. Except for particles in the first quarter wavolength of the bunch, all particles experience the aame retarding potential. At the end of the doorstep $(N=1 / 4), R=\sqrt{2}$ and $\eta=2 / \pi$. 
As a numerical example, consider an accelerator operating at $\lambda=1 \mathrm{~cm}$ with a desired gradient of $200 \mathrm{mV} / \mathrm{m}$. A SLAC-type atructure at this wavlength would have a loss parameter on the order $2 \times 10^{15} \mathrm{~V} / \mathrm{C}-\mathrm{m}$. With a transformer ratio of 20, driving burehes with an energy of $100 \mathrm{MeV}$ would nied to be injected every ten meters. The charge per bunch as given by Eq. (a) is

$$
g=\frac{E_{a} R}{4 k \eta} \approx 0.5 \mu \mathrm{C}
$$

assuming that most of the energy goes into a single mode and that the efficiency is clase to $100 \%$. The bunch length is epproximately $R \lambda / 2 \pi=3.2 \mathrm{~cm}$ or $100 \mathrm{ps}$, and the peak current at the end of the bunch is $10 \mathrm{kA}$. Many practical questions must be addrassed, such as the feasibility of creating properily shaped bunches with very high peak currents. The deflecting Gelds induced if the driving bunch wanders off the axis of the structure are also a serious problem.

\section{Ring Beams in Cylindrically Symmetric Structures}

In this section we consider cylindrically symrietric structures excited by a beam in the form of a ring, or hollow cylinder with thin "walls": A simple case for which the beam-structure interaction can be computed analytically is the pillbox cavity having a thin arimuthal slot at radius ro, as shown in Fig. 12. If the slot is located near the outer perimeter of the cavity, we might expect a large transformer ratio. In qualitative terms, a driving bunch entering the cavity generates a wave packet which travels towerd the axis. The volumn of the wave packet decrease roughly as $r^{-1}$, and the electric and magnetic field strengths must therefore increase approximately as $r^{-1 / 2}$. A second bunch can then be accelarated along the axis of the cavity. In a practical structure, the effect of the axjal beam tube and the azimuthal slot on the wake potentials must be taken into account.

The advantage of a ring beam over a line beam at the same radius is that higher order azimuthal modes $(m>0)$ are not excited. Since a ring bearn

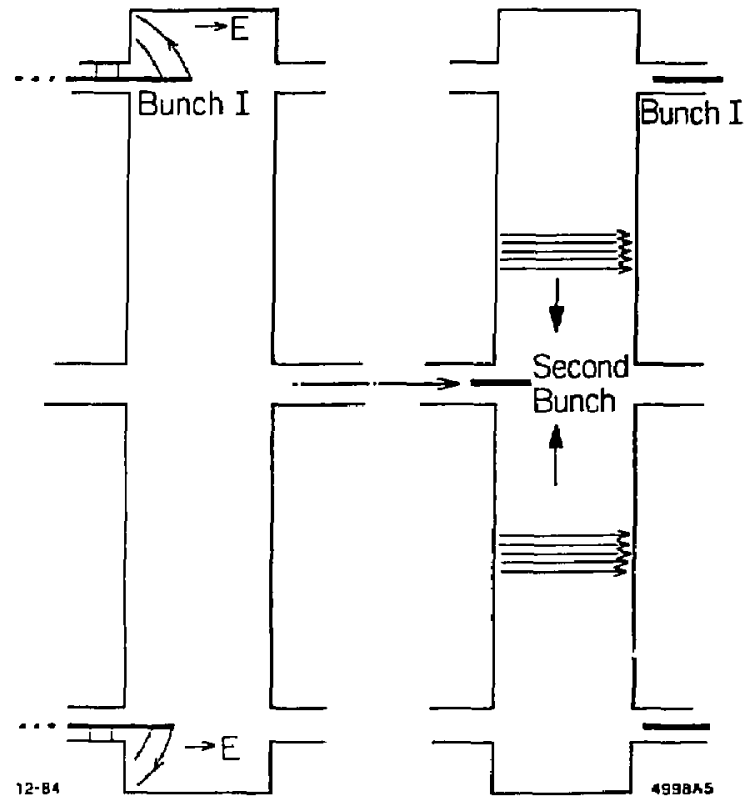

Figure 12. Qualitative picture of the field induced by a ring bunch passing through a pillbox cavity. 
is a superposition of an infinite number of line beams at the same radius, the transformer ratio for a ring beam with radius ro and a line beam at radius $r_{0}$ with the same axial charge distribution would be the same if azinuthally varying modes ere ignored. Inclusion of these modes would degrade the transformer ratio, and in addition a deflecting wake would be gencrated on the axis of the pillbox.

If only the lowest frequency TMp1o mode in a pillbox cavity is excited by the ring driving bunch, the iransformer ratio for a ring bunch with axial extent $\Delta z=0$ can be abtained from Eq. (20a) as

$$
\quad R=2\left[\frac{k(0)}{k\left(r_{0}\right)}\right]^{1 / 2}=\frac{2}{J_{0}\left(\text { pat } r_{0} / b\right)}
$$$$
\text { . . . it. }
$$

where $b$ is the outer radius of the cavity and $p_{0}=2.405$ is the first root of $J_{0}$. In practice, a lone buncii exciles predominately the lowest frequency mode. For a Hat current distribution (rectangular bunch) of length $\Delta z$, Eqs. (3ta) and (31b) can be used to show that the above transformer ratio must be multiplied by a bunch form factor

$$
\begin{array}{ll}
F=\frac{\sin \left(\pi \Delta z / \lambda_{0}\right)}{\sin \left(2 \pi \Delta z / \lambda_{0}\right)} ; & \frac{\Delta z}{\lambda_{0}}<\frac{1}{4} \\
F=\sin \left(\pi \Delta z / \lambda_{0}\right), & \frac{\Delta z}{\lambda_{0}}>\frac{1}{4}
\end{array}
$$

where $\lambda_{0}$ is the wavelength of the fundamental mode. Mitrovich et al ${ }^{13}$ have computed the energy going into higher mades for a pillbox cavity with $g / \lambda_{0}=0.1$ and $\Delta z / \lambda_{0}=0.425$ as a function of the beam radius $r_{0}$. For this driving bunch length, more than $90 \%$ of total energy deposited in all modes goes into the fundamental mode for $0.15<r_{0} / b<0.72$. At the upper end of this range, the transformer ratio is 5.1. Higher transiormer ratios could of course be reached by making ro/b eloser to one. However, more encrgy then goes into higher modes. In addition, the driving bunch distribution could be shaped, as discussed in the previous section.
A wake field "transformer," which consists essentially of a serieg of pillbox cavitieg with a ring gap near the outer radius and a hole on the axis for the axcelerated beam, has been propused by Yoss and Weiland. ${ }^{14,15}$ A12 experiment ${ }^{16,17}$ is in progress at DESY to test the concept by injecting an $8 \mathrm{MieV}, 1 \mu \mathrm{C}$ beam into the structure shows schematically in Fig. 13. Also shown are the wake fields calculated at eight time steps by T. Weiland using his code TBCI. ${ }^{\text {I8 }}$ Nate that the outer boundary of the structure has been shaped to enhance the transfer of energy into the radially propagating wake fields. After the wave teaches the axis it is reflected and travels back to the outer boundary. There it is refected once again, travels back to the axis, and produces a second high field pulse. This second pulse has the inverse sign and can be used to accelerate positrons.

Figure If shows the longitudinal potential due to these fields for the driviag beam and for an accelerated bearn on the axis. The maximum decelerating wake potential seen by the particles in the driving beasd is computed to be $17 \mathrm{mV} / \mathrm{m}$, but on the axis a gradient of $170 \mathrm{mV} / \mathrm{m}$ is produced. Thus the transformer ratio for this particular structure is 10 .

Some important observations can be made concerning the deflecting wake fields in the driving beam. For the usual cavity with no metal between the beam and the axis, recall that the dipole wake potential can be written as

$$
\text { e } \int \mathbf{F} d l=\hat{\mathbf{r}} W_{\mathrm{r}}(s) \cos \phi+\hat{\phi} W_{\phi}(s) \sin \phi=W_{\perp}(s)(\hat{r} \cos \phi-\hat{\phi} \sin \phi),
$$

where $W_{r}(s)=-W_{\phi}(s)$. We now find, when there is metal between a hollow beam and the axis, that the deflecting forces cannot be described by a single transwerse wake function. ${ }^{18}$ Two functions are now required at a given fixed radius for the ring beam (and four if the position of the ring beam is allowed to vary):

$$
c \int F_{\perp}(r, \phi) d t=f W_{r}(s) \cos \phi+\hat{\phi} W_{\phi}(s) \sin \phi .
$$

where $W_{r}(s) \neq-W_{\phi}(s)$. hs an example, Fig. 15 shows the dipole walke potentials for the inner bunch $\left(W_{r}=W_{\phi}\right)$ and the outer bunch $\left(K_{r} \neq-W_{\phi}\right)$ for the 

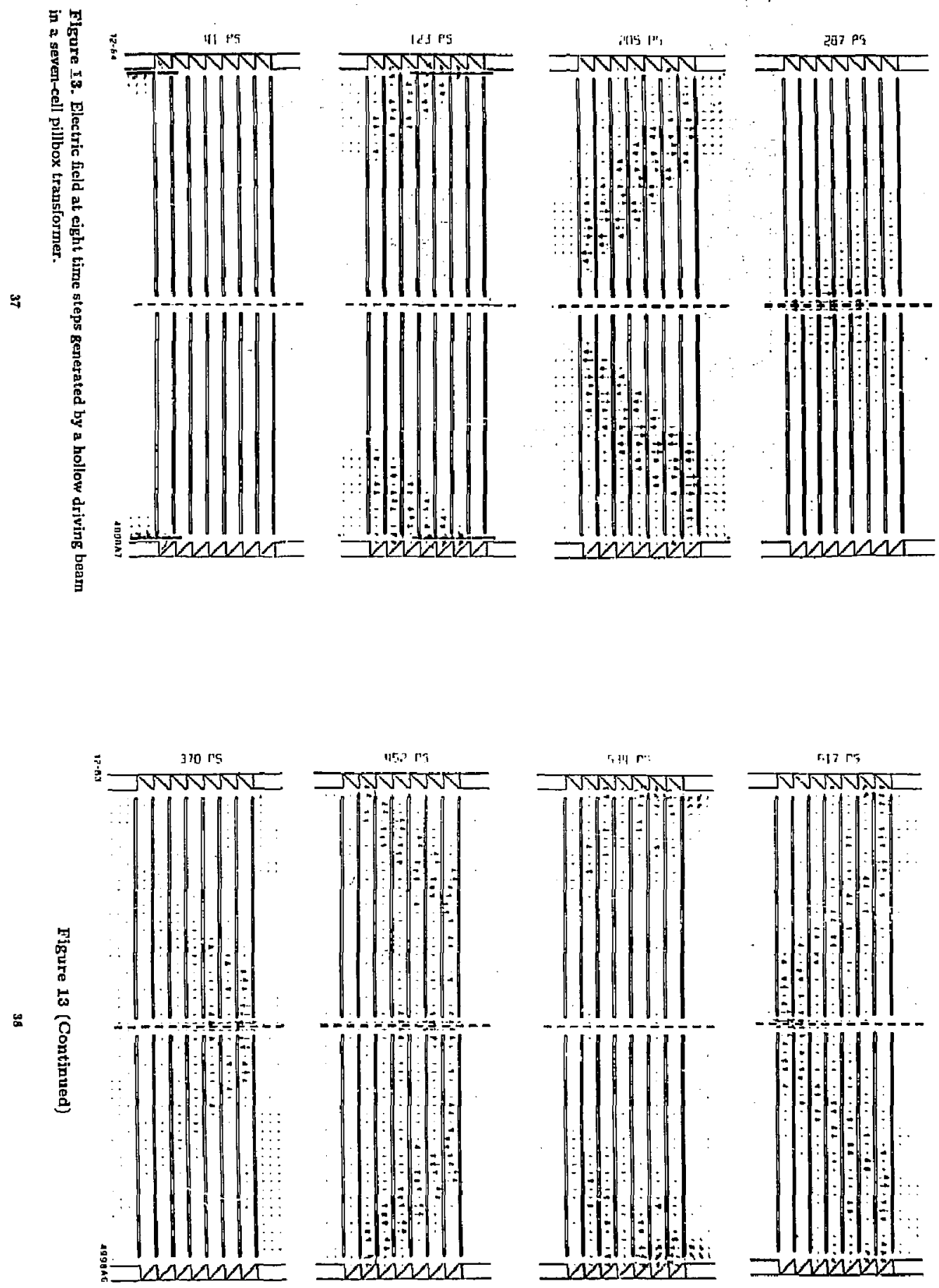


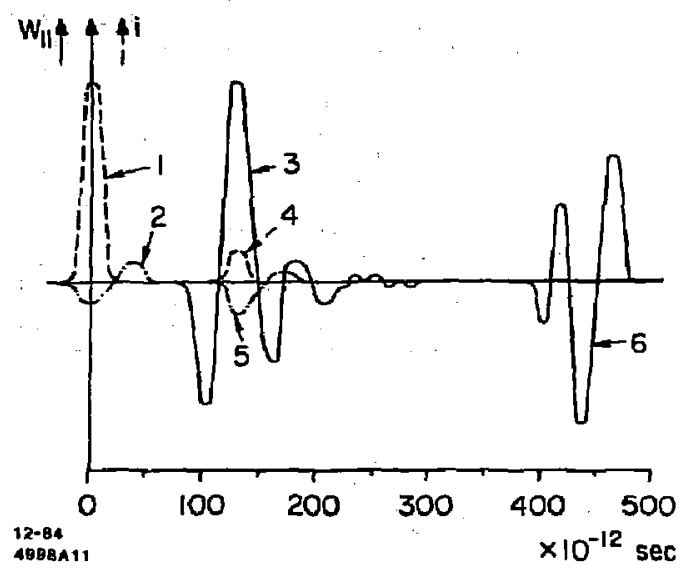

Fignre 14. Longitudinal potential for the wake field transformer shown in Fig. 13. (1) Driving beam density; (2) decelerating potential inside driving beam; (3) eccelerating polential ou axis; (4) density of the accelerated beam; (5) self potential of the accelerated beam; (6) accelerating potential after refiection from outer wall.
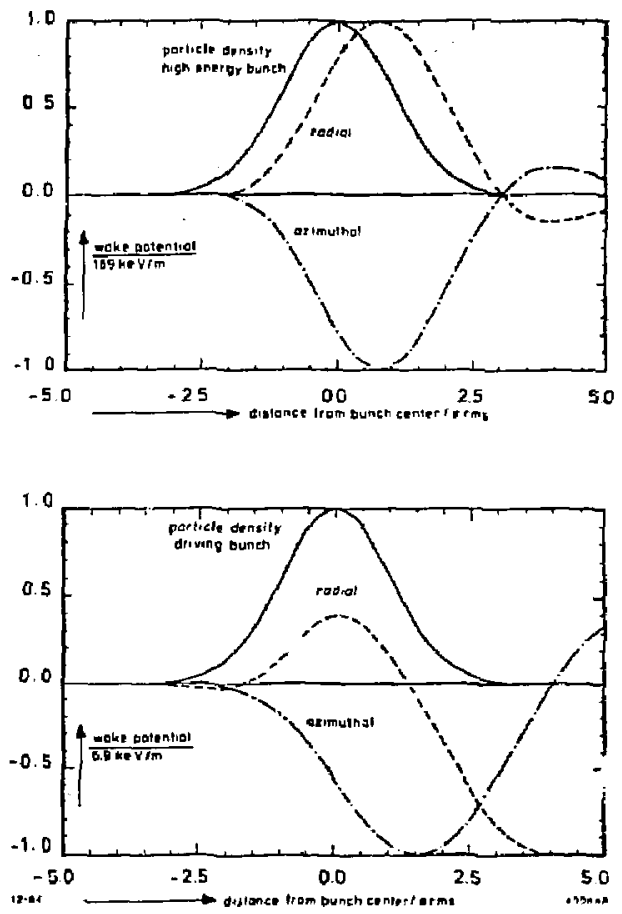

Figure 15. Transverse wake potential on the axis (top) and at the outer driving ring heam (bottom) generated by a $0.1 \mathrm{~mm}$ offset of the driving beam in the structure shown in Fig. 13. The parameters of the driving beam are $\sigma_{x}=2 \mathrm{~mm}$, $Q=1 \mu C$ and $r_{0}=5 \mathrm{~cm}$. 


\section{REFERENCES}

1. R.D. Ruth and Pisin Chen, Plasmo slceleyators, these Proceedings. Also SLAC-PUB-3906, March 1986.

2. K. Satoh, private communication.

3. A.W. Chao, PEP-Note-332, SLAC, June 1980.

4. A.W. Chao and P.L. Morton, PEP-Note-105, SLAC, 1975.

5. K. Bane, private communication. See also T. Weiland and B. Zotter, Part. Accal. 11, 143 (198I).

6. K.L.F. Bane, P.B. Wilson and T. Weiland in Phyries of Migh Energy Particle Accelerators, BNL/SUNY Summer School, 1983, M. Month, P. Dahl and M. Dienes, cds, AIP Conference Procecdings No. 127 (American Institute of Physics, New York, 1985), pp. 875-928. Also SLAC-PUB-3528, December 1984.

7. K. Bane, CEHN/ISR-TH/B-47, 1980.

8. W.K.H. Panofsky and W.A. Wenzel, Rev. Sci. Instrum. 27, 967 (1956).

9. P.B. Wilson in Physics of Bigh Energy Particle Accelerators, Fermilab Summer School, 1981, R.A. Carrigan, F.R. Huson, M. Month, eds., AIP Confercnce Proceedings No. 87 (American Institute of Physies, Now York, 1982). Sec. 6.1. Also SLAC-PUB-2884, February 1982.

10. See for example R.E. Collin, Fourdations for Microwaut Enginetring (McGraw-Hill, New York, 1966), Sec. 2.12.

11. K. Bane and P.B. Wilson, Proceedings of the 11th International Gonference on High-Energy Accelerators, CERN, July 1980 (Birkhäuser Verlag, Basel, 1980), p. 592.

12. K.L.F. Bane, Pisin Chen and P.B. Wileon, IEEE Trans. Nucl. Sci. NS-32, No. 5, 3524 (1985). Also SLAC-PUB-3662. 
13. D. Mitravich it ed., IFas Trans, Nucl. Sci. NS-30, No. 4, 3518 (1983).

14. G.A. Voes and T. Weiland, DESY M-82-10, April 1982.

15. G.A. Voes and T. Weiland, DESY 82-074, Novemaber 1982.

16. The Wake Field Accelerator Study Group, DESY M-83-27, 1983; and Proecedings of the Ieth foternational Confarenee on High Energy Aceclerators, Fermilnb, 1083, pi 454,

17. T. Weiland, IFEE Trams. Nucl. Sci. NS-39, No. 5, 3471 (1985).

18. T. Wellánd, DESY E2-015, 1982; and Nucl. Instruma. Methods 212, 131 (1983).

19. 5. Takeda, Osaks Univerdity, private communication.

20. Y. Chin, KEK 83-19, 1983. 